\title{
A refined analysis of the remarkable Bp star HR $6000^{\star, \star \star}$
}

\author{
F. Castelli ${ }^{1}$ and S. Hubrig ${ }^{2}$ \\ 1 Istituto Nazionale di Astrofisica-Osservatorio Astronomico di Trieste, via Tiepolo 11, 34131 Trieste, Italy \\ e-mail: castelli@oats.inaf.it \\ 2 European Southern Observatory, Casilla 19001, Santiago 19, Chile \\ e-mail: shubrig@eso.org
}

Received 22 May 2007 / Accepted 5 September 2007

ABSTRACT

\begin{abstract}
Aims. UVES spectra of the very young ( $10^{7}$ years $)$ peculiar B-type star HR 6000 were analyzed in the near-UV and visual spectral regions (3050-9460 $\AA$ ) with the aim of extending to other spectral ranges the study made previously in the UV using IUE spectra. Methods. Stellar parameters $T_{\text {eff }}=12850 \mathrm{~K}, \log g=4.10$, and $\xi=0 \mathrm{~km} \mathrm{~s}^{-1}$, as determined from $\mathrm{H}_{\beta}, \mathrm{H}_{\gamma}, \mathrm{H}_{\delta}$ Balmer profiles and from the Fe I, Fe II ionization equilibrium, were used to compute an individual abundances ATLAS12 model. We identified spectral peculiarities and obtained final stellar abundances by comparing observed and computed equivalent widths and line profiles.

Results. The adopted model fails to reproduce the $(b-y)$ and $c$ color indices. The spectral analysis revealed: the presence of emission lines for Mn II, Cr II, and Fe II; isotopic anomalies for $\mathrm{Hg}, \mathrm{Ca}$; the presence of interstellar lines of $\mathrm{Na}$ I at $\lambda \lambda$ 3302.3, 3302.9, 5890, $5896 \AA$, and of $\mathrm{KI}$ at $7665,7699 \AA$; the presence of a huge quantity of unidentified lines, which we presume to be mostly due to Fe II transitions owing to the large Fe overabundance amounting to [+0.7]. The main chemical peculiarities are an extreme overabundance of $\mathrm{Xe}$, followed by those of $\mathrm{Hg}, \mathrm{P}, \mathrm{Y}, \mathrm{Mn}, \mathrm{Fe}, \mathrm{Be}$, and Ti. The most underabundant element is Si, followed by $\mathrm{C}, \mathrm{N}$, $\mathrm{Al}, \mathrm{S}, \mathrm{Mg}, \mathrm{V}, \mathrm{Sr}, \mathrm{Co}, \mathrm{Cl}, \mathrm{Sc}$, and $\mathrm{Ni}$. The silicon underabundance [-2.9] is the lowest value for Si ever observed in any $\mathrm{HgMn}$ star. The observed lines of He I cannot be reproduced by a single value of the He abundance, but they require values ranging from [ -0.8 ] to [-1.6]. Furthermore, when the observed and computed wings of He I lines are fitted, the observed line cores are much weaker than the computed ones. From the present analysis we infer the presence of vertical abundance stratification for He, Mn, and possibly also Fe and $\mathrm{P}$.
\end{abstract}

Key words. stars: abundances - line: identification - atomic data - stars: atmospheres - stars: chemically peculiar stars: individual: HR $6000(\mathrm{Bp})$

\section{Introduction}

HR 6000 (HD 144667) is one of the most remarkable chemically peculiar (CP) stars. It does not fit any of the $\mathrm{CP}$ subclasses, but seems to combine abundance anomalies from a variety of Bp sub-types. It forms with the star HR 5999 (HD 144668) the common proper motion visual binary system $\Delta 199$ or Dunlop 199 (Bessell \& Eggen 1972). The angular separation between the HR 6000, which is the brighter more massive component, and the secondary component, the well-known Herbig Ae star HR 5999, is about 45".

The main interest in studing the chemical composition of HR 6000 comes from the generally poor understanding of the occurrence of abundance anomalies in such a young object with an estimated age of the order of $10^{7}$ years. In fact, the $\Delta 199$ double system is located close to the center of the Lupus 3 molecular cloud which is populated by numerous T Tauri stars. This has led to an assumption that this system has the same age as the cloud, which is estimated to be $(9.1 \pm 2.1) \times 10^{6}$ years (James et al.2006). However, after the Hipparcos mission, the membership of HR 5999 and HR 6000 to the cloud became rather questionable due to the uncertainties in the distance determination for the Lupus cloud. The distance of HR 5999 and HR 6000

* Based on observations collected at the European Southern Observatory, Paranal, Chile (ESO programme 076.D-0169(A)).

$\star \star$ Table 6 is only available in electronic form at http://www. aanda.org measured by Hipparcos are $208 \pm 38$ pc and $240 \pm 48$ pc, respectively, while the Lupus cloud distance is $150 \pm 10 \mathrm{pc}$ according to Crawford (2000). Comerón et al. (2003) assigned a distance of about $200 \mathrm{pc}$ to the Lupus cloud, but this determination was made by assuming a priori that $\Delta 199$ belongs to the cloud.

A ROSAT survey of Herbig $\mathrm{Ae} / \mathrm{Be}$ stars presented by Zinnecker \& Preibisch (1994) detected strong X-ray emission coming from the direction of HR 6000. To explain the X-ray origin, a possible T Tauri companion for HR 6000 was postulated by van den Ancker et al. (1996) on the basis of an infrared excess in the energy distribution relative to predictions made for an effective temperature of $T_{\text {eff }}=14000 \mathrm{~K}$. Siebenmorgen et al. (2000) fitted very short spectral regions of observed flux in the mid IR to a black body of $13000 \mathrm{~K}$ and noticed that the observed spectrum was featureless.

Neither spectrum nor radial velocity variability was found for HR 6000 by Andersen \& Jaschek (1984), who studied optical spectra taken at different epochs. However, van den Ancker et al. (1996) discovered long-term variations in the $u, v$, and $b$ Strömgren magnitudes with approximate amplitude of $0.03 \pm 0.01$ in $u, 0.02 \pm 0.01$ in $v$, and 0.01 in $b$. No variations in $y$ were found. Kurtz \& Marang (1995) discovered variations of $0.008 \mathrm{mag}$ in $V$ with a period near to $2 \mathrm{~d}$ and suggested that this period could be possibly caused by rotational variation in a spotted magnetic star. This would imply a pole on orientation for HR 6000 as they measured $v \sin i \leq 5 \mathrm{~km} \mathrm{~s}^{-1}$. 
Table 1. Stellar parameters for HR 6000 from photometry by assuming $[\mathrm{M} / \mathrm{H}]=0.0$ and $\xi=0.0 \mathrm{~km} \mathrm{~s}^{-1}$.

\begin{tabular}{|c|c|c|c|c|c|c|c|c|c|c|}
\hline \multicolumn{5}{|c|}{ Observed indices } & \multicolumn{3}{|c|}{ Dereddened indices } & \multicolumn{2}{|c|}{ Parameters } & \multirow[b]{2}{*}{$E(B-V)$} \\
\hline$(b-y)$ & $m_{1}$ & $c_{1}$ & $\beta$ & $E(b-y)$ & $(b-y)_{0}$ & $m_{0}$ & $c_{0}$ & $T_{\text {eff }}(\mathrm{K})$ & $\log g$ & \\
\hline \multirow{4}{*}{$\begin{array}{l}-0.030 \\
\pm 0.003\end{array}$} & \multirow{4}{*}{$\begin{array}{l}0.116 \\
\pm 0.003\end{array}$} & \multirow{4}{*}{$\begin{array}{l}0.512 \\
\pm 0.003\end{array}$} & \multirow{4}{*}{$\begin{array}{l}2.750 \\
\pm 0.013\end{array}$} & \multirow[t]{2}{*}{0.031} & \multirow[t]{2}{*}{-0.061} & \multirow[t]{2}{*}{0.126} & \multirow[t]{2}{*}{0.506} & 13799 & 4.27 & \multirow[t]{2}{*}{0.042} \\
\hline & & & & & & & & \pm 150 & \pm 0.05 & \\
\hline & & & & \multirow[t]{2}{*}{0.044} & \multirow[t]{2}{*}{-0.074} & \multirow[t]{2}{*}{0.130} & \multirow[t]{2}{*}{0.503} & 13827 & 4.27 & \multirow[t]{2}{*}{0.060} \\
\hline & & & & & & & & \pm 200 & \pm 0.05 & \\
\hline $\bar{V}$ & $U-B$ & $B-V$ & & $E(B-V)$ & $\overline{V_{0}}$ & $(U-B)_{0}$ & $(B-V)_{0}$ & $T_{\text {eff }}(\mathrm{K})$ & $\log g$ & \\
\hline 6.647 & -0.434 & -0.073 & & 0.06 & 6.461 & -0.477 & -0.133 & 13973 & 4.38 & \\
\hline \pm 0.013 & \pm 0.004 & \pm 0.004 & & & & & & \pm 300 & \pm 0.2 & \\
\hline & $(V-I)_{\mathrm{c}}$ & $(V-J)$ & & $E\left(V-I_{\mathrm{c}}\right)$ & $E(V-J)$ & $\left(V-I_{\mathrm{c}}\right)_{0}$ & $(V-J)_{0}$ & $T_{\text {eff }}(V-J)$ & $T_{\text {eff }}\left(V-I_{\mathrm{c}}\right)$ & $\log g$ \\
\hline & $\begin{array}{l}-0.05 \\
\pm 0.00\end{array}$ & -0.032 & & 0.075 & 0.134 & -0.125 & -0.166 & 11517 & 10889 & 4.0 (prefixed) \\
\hline model & & & & & $(b-y)_{\text {calc }}$ & & $c_{\text {calc }}$ & & & \\
\hline T12950 & $405 \mathrm{k} 2 \mathrm{M} 0$ & ohe & & & -0.041 & & 0.629 & & & \\
\hline $\mathrm{T} 12850$ & 1k0at12 & & & & -0.049 & & 0.596 & & & \\
\hline
\end{tabular}

Catanzaro et al. (2004) were the first to provide abundances from the optical range. Previously, optical spectra had only been studied by Andersen \& Jaschek (1984) and Andersen et al. (1984) who identified the spectra from $3323 \AA$ to $5317 \AA$ and discussed the abundances on the basis of the line intensities. It is remarkable that the stellar parameters derived by Catanzaro et al. (2004) from the Balmer profiles $\left(T_{\text {eff }}=12950 \pm 50 \mathrm{~K}, \log g=4.05 \pm 0.01\right)$ are $1000 \mathrm{~K}$ lower than those previously adopted and deduced from the photometry by Castelli et al. (1985) $\left(T_{\text {eff }}=14000 \mathrm{~K}, \log g=4.0\right)$, by Smith (1997) $\left(T_{\mathrm{eff}}=13990 \mathrm{~K}, \log g=4.29\right)$ and by van den Ancker et al. (1996) $\left(T_{\text {eff }}=14000 \mathrm{~K}, \log g=4.3\right)$.

In this paper we examine the whole optical spectrum of HR 6000 from $3050 \AA$ to $9460 \AA$ with the aim of extending the analysis performed on IUE spectra by Castelli et al. (1985) to the visible region and to investigate the possible contamination of the stellar spectrum by a close T Tau companion. We reexamine the parameter determination, the line identification, and the abundances. We also searched for the presence of peculiarities such as emission lines and isotopic anomalies which were recently discovered in $\mathrm{HgMn}$ stars. Owing to the availability of spectra taken at different epochs we also investigated possible spectral variabilities.

The comparison of the observed and computed spectra as described in this paper is available at the web address given in the footnote ${ }^{1}$.

\section{Observations and radial velocity}

Spectra of HR 6000 were recorded at ESO on 2005 September 19 (JD 2453632.53881) and on 2006 March 25 (JD 2453 819.90226). In both epochs we used the UVES DIC1 and DIC2 standard settings to cover the spectral range from $3050 \AA$ to $9500 \AA$. The slit width was set to 0.3 for the red arm, corresponding to a resolving power of $\lambda / \Delta \lambda \approx 110000$. For the blue arm, we used a slit width of $0 . ' 4$ to achieve a resolving power of $\approx 80000$. The spectra were reduced by the UVES pipeline Data Reduction Software (version 2.5; Ballester et al. 2000) and using standard IRAF routines. The signal-to-noise ratio $(\mathrm{S} / \mathrm{N})$ of the UVES spectra is very high, ranging from 150

\footnotetext{
1 http://wwwuser.oat.ts.astro.it/castelli/ hr6000/hr6000.html
}

in the near UV (3300 $\AA$ ) to about 350 and 300 at $5000 \AA$ and $7100 \AA$, respectively.

There are two gaps in the observed range at $\lambda \lambda 4523-4769 \AA$ and 7536-7660 $\AA$, which are caused by the physical gap between the two detector chips of the red CCD mosaic. In addition, the reduction in the spectral range $8076-8093 \AA$ is affected by the presence of a bad column on the MIT CCD.

The heliocentric radial velocities derived from spectra observed on September 2005 and on March 2006 are $2.6 \mathrm{~km} \mathrm{~s}^{-1}$ and $1.4 \mathrm{~km} \mathrm{~s}^{-1}$, respectively. These values are consistent with the mean heliocentric radial velocity $+2.6 \pm 1.8 \mathrm{~km} \mathrm{~s}^{-1}$ of the Lupus cloud (James et al. 2006), so that they strenghten the assumption of the membership of HR 6000 to the Lupus cloud. However, Catanzaro et al. (2004) measured $0.67 \pm 0.38 \mathrm{~km} \mathrm{~s}^{-1}$, while Andersen \& Jaschek (1984) determined $-1.5 \pm 0.5 \mathrm{~km} \mathrm{~s}^{-1}$. In their study, the values obtained from different spectra, range from $+0.6 \pm 0.8 \mathrm{~km} \mathrm{~s}^{-1}$ to $-3.3 \pm 0.8 \mathrm{~km} \mathrm{~s}^{-1}$. Such differences in measured radial velocities by different authors indicate that small radial velocity variations in HR 6000 are possibly real.

The spectra were normalized to the continuum by using a modified version of the interactive code NORMA written by Bonifacio (1989). In the original procedure smoothed interpolated curves through selected continuum points are obtained by means of Hermite spline functions (subroutine INTEP by Hill 1982). We replaced the spline functions with a linear interpolation because in some previous stellar analyses that we performed it has given a continuum closer to what we would have drawn by hand. However, we checked that in the case of HR 6000 the continua from the two different interpolations do not differ significantly. When needed, the continuum was adjusted over $6 \AA$ intervals with the help of the synthetic spectrum. The continuous level just longward of the Balmer and Paschen discontinuities is highly uncertain because it is difficult to know where to put it. Also the continuum drawn over the broad hydrogen lines is uncertain owing to the incompletely corrected distorsions of the echelle spectra.

\section{Stellar parameters}

Table 1 summarizes the stellar parameters as determined from Strömgren, UBV, and near-infrared IJ photometry. The observed Strömgren and UBV indices were taken from the 
Hauck \& Mermilliod (1998) Catalogue ${ }^{2}$, while $\left(V-I_{\mathrm{c}}\right)=-0.05$ was taken from the Hipparcos Catalogue and $(V-J)=-0.032$ was obtained from the $J$ magnitude given by Thé et al. (1996). The parameter determination is the result of an interpolation of the observed dereddened indices in the grid of synthetic indices based on the NEWODF model atmospheres (Castelli \& Kurucz 2004) computed for metallicity $[\mathrm{M} / \mathrm{H}]=0.0$ and microturbulent velocity $\xi=0.0 \mathrm{~km} \mathrm{~s}^{-1}$. The color grids are available at the web address given in the footnote ${ }^{3}$. For the Strömgren and UBV photometry the adopted grids were uvby $\beta$ and UBV, while for the $\left(V-I_{\mathrm{c}}\right)$ and $(V-J)$ indices, the adopted grid was UBVRIJHKL.

Results from the Strömgren photometry are listed in the first panel of Table 1 . For $E(B-V)=0.06$, the parameters from the $\mathrm{c}_{0}$ and $\beta$ indices are $T_{\text {eff }}=13827 \pm 200 \mathrm{~K}, \log g=4.27 \pm 0.05$. The reddening $E(B-V)=0.06$ was determined by Bessell \& Eggen (1972) and later confirmed by Thé \& Tjin A Djie (1978). An uncertainty in $E(B-V)$ of 0.02 mag corresponds to a difference in $T_{\text {eff }}$ of only $15 \mathrm{~K}$. The reddening $E(B-V)=0.042$ listed in the first row of Table 1 corresponds to $E(b-y)=0.031$ as yielded by the UBVYLIST code of Moon (1985) on the basis of standard reddening relations. The conversion $E(b-y)=0.74 E(B-V)$ from Crawford \& Mendwewala (1976) was applied here.

A microturbulent velocity of $2.0 \mathrm{~km} \mathrm{~s}^{-1}$ lowers the temperature by $100 \mathrm{~K}$ and does not affect the gravity, while an increase in metallicity to $[\mathrm{M} / \mathrm{H}]=+0.5$ decreases $T_{\text {eff }}$ by about $50 \mathrm{~K}$; a decrease in metallicity to $[\mathrm{M} / \mathrm{H}]=-1.0$ increases $T_{\text {eff }}$ by about $+300 \mathrm{~K}$. The corresponding differences in $\log g$ are of the order of 0.01 dex in all cases. These values are only indicative of the metallicity effect in that the abundance pattern of HR 6000 is very different from any scaled solar patterns.

The parameters from the UBV photometry and $\beta$ index are listed in the second panel of Table 1. For $E(B-V)=0.06$, $[\mathrm{M} / \mathrm{H}]=0.0$, and $\xi=0.0 \mathrm{~km} \mathrm{~s}^{-1}$ they are $T_{\text {eff }}=13973 \pm 300 \mathrm{~K}$, $\log g=4.38 \pm 0.2$ (Fig. 1). These values are close to those obtained from the Strömgren photometry. This parameter determination, described in Castelli et al. (1985), is very sensitive to small reddening changes. In fact, for $E(B-V)=0.04$ or $E(B-V)=0.08$ there is no intersection in the curves $(U-B)_{0}$, $(B-V)_{0}$ and $\beta$. For B-type stars, the $(U-B),(B-V)$, and $\beta$ indices do not depend on the metallicity significantly (Castelli 1999; Castelli \& Kurucz 2006).

The third panel of Table 1 shows that for a prefixed gravity $\log g=4.0$, the observed $(V-I)_{\mathrm{c}}$ and $(V-J)$ indices are reproduced by $T_{\text {eff }}$ equal to $10889 \mathrm{~K}$ and $11517 \mathrm{~K}$, respectively, when $[\mathrm{M} / \mathrm{H}]=0.0, \xi=0.0 \mathrm{~km} \mathrm{~s}^{-1}$, and $E(B-V)=0.06$. We used the reddening relations $E\left(V-I_{\mathrm{c}}\right)=1.25 E(B-V)$ from Dean et al. (1978) and $E(V-J)=2.23 E(B-V)$ from Mathis (1990). The temperature was derived by interpolating the observed dereddened $\left(V-I_{\mathrm{c}}\right)_{0}$ and $(V-J)_{0}$ indices in the Bessell et al. (1998) color grid computed for $[\mathrm{M} / \mathrm{H}]=0.0$ and $\xi=0.0 \mathrm{~km} \mathrm{~s}^{-1}$.

Catanzaro et al. (2004) were the first to derive parameters for HR 6000 from high resolution spectra, in particular from $\mathrm{H}_{\gamma}$ and $\mathrm{H}_{\delta}$ Balmer profiles observed on FEROS spectra. They fixed $T_{\text {eff }}=12950 \pm 50 \mathrm{~K}$ and $\log g=4.05 \pm 0.01$ from ATLAS9 models computed for $[\mathrm{M} / \mathrm{H}]=-0.5$ and zero helium abundance. This particular grid of models yields the same parameters from both Balmer profiles, but we note that the parameters do not change if the grid computed with $[\mathrm{M} / \mathrm{H}]=0.0$ and solar He abundance is used, provided that the different parameters from $\mathrm{H}_{\gamma}$ ([12750 K, 3.95] and $\mathrm{H}_{\delta}([13150 \mathrm{~K}, 4.13])$ are averaged. As

\footnotetext{
2 http://www. unige.ch/gcpd/gcpd.html

3 http://wwwuser . oat.ts.astro.it/castelli/colors.html
}

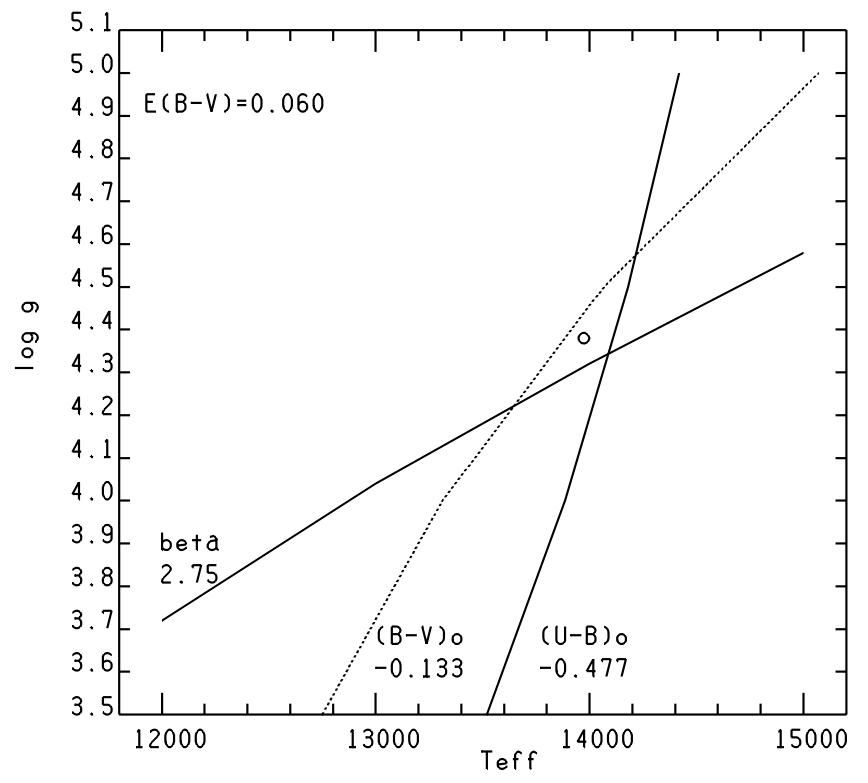

Fig. 1. Determination of the atmospheric parameters $\left(T_{\text {eff }}, \log g\right)$ from $(U-B)_{0},(B-V)_{0}$ and $\beta$. The circle represents the parameters $T_{\text {eff }}=13873 \mathrm{~K}, \log g=4.38$ obtained from this method for $[\mathrm{M} / \mathrm{H}]=0.0$ and $\xi=0.0 \mathrm{~km} \mathrm{~s}^{-1}$. The observed indices were dereddened for $E(B-V)=0.060$.

discussed in Sect. 4, we derived parameters very close to those of Catanzaro et al. (2004) when we used Balmer profiles, Fe I, Fe II equivalent widths measured on UVES spectra, and model atmospheres computed for the individual abundances of HR 6000.

The last panel of Table 1 shows that the cooler model, as adopted by Catanzaro et al. (2004) (model T12950g405k2M05nohe), does not reproduce the observed Strömgren indices. In fact, the difference between the observed and computed $(b-y)_{0}$ indices amounts to $0.020 \mathrm{mag}$, or even more, depending on the adopted reddening, while the difference between the $c_{0}$ indices is $0.122 \mathrm{mag}$. These discrepancies do not change remarkably for the ATLAS12 model (model T12850g41k0at12) we adopted to predict the observed spectrum (see Sect. 4). On the other hand, the hotter model with parameters from photometry [13900, 4.32] fails to reproduce all Balmer profiles. For solar abundances it gives good agreement between observations and computations for $\mathrm{H}_{\alpha}$, but the agreement decreases from $\mathrm{H}_{\beta}$ to $\mathrm{H}_{\delta}$ in the sense that the computed wings become too broad as compared with those observed; for individual abundances the computed inner wings of all the profiles are narrower than those observed, in particular for $\mathrm{H}_{\alpha}$ whose profile seems also to be slightly asymmetric with a more extended red wing. All comparisons between the observed and computed Balmer profiles are available at the website of footnote 1 . Figure 2 shows the case of $\mathrm{H}_{\beta}$. In conclusion, there is a discrepancy of about $1000 \mathrm{~K}$ between parameters derived from UBV and Strömgren photometry, and parameters derived from the line spectrum. Furthermore, the difference in $T_{\text {eff }}$ from the near-IR photometry is so large that spectrophotometric observations are needed to confirm these results. Unfortunately, the only spectrophotometric data available for HR 6000 are the IUE spectra. For $E(B-V)=0.06$, the best fit of the UV energy distribution from the IUE images SWP14849 and LWR11431 to the ATLAS9 energy distributions computed for $[\mathrm{M} / \mathrm{H}]=0.0$ and $\xi=0.0 \mathrm{~km} \mathrm{~s}^{-1}$, is achieved with $T_{\text {eff }}=13300 \mathrm{~K}, \log g=4.1$. More details about these IUE images can be found in Thé et al. (1996). 

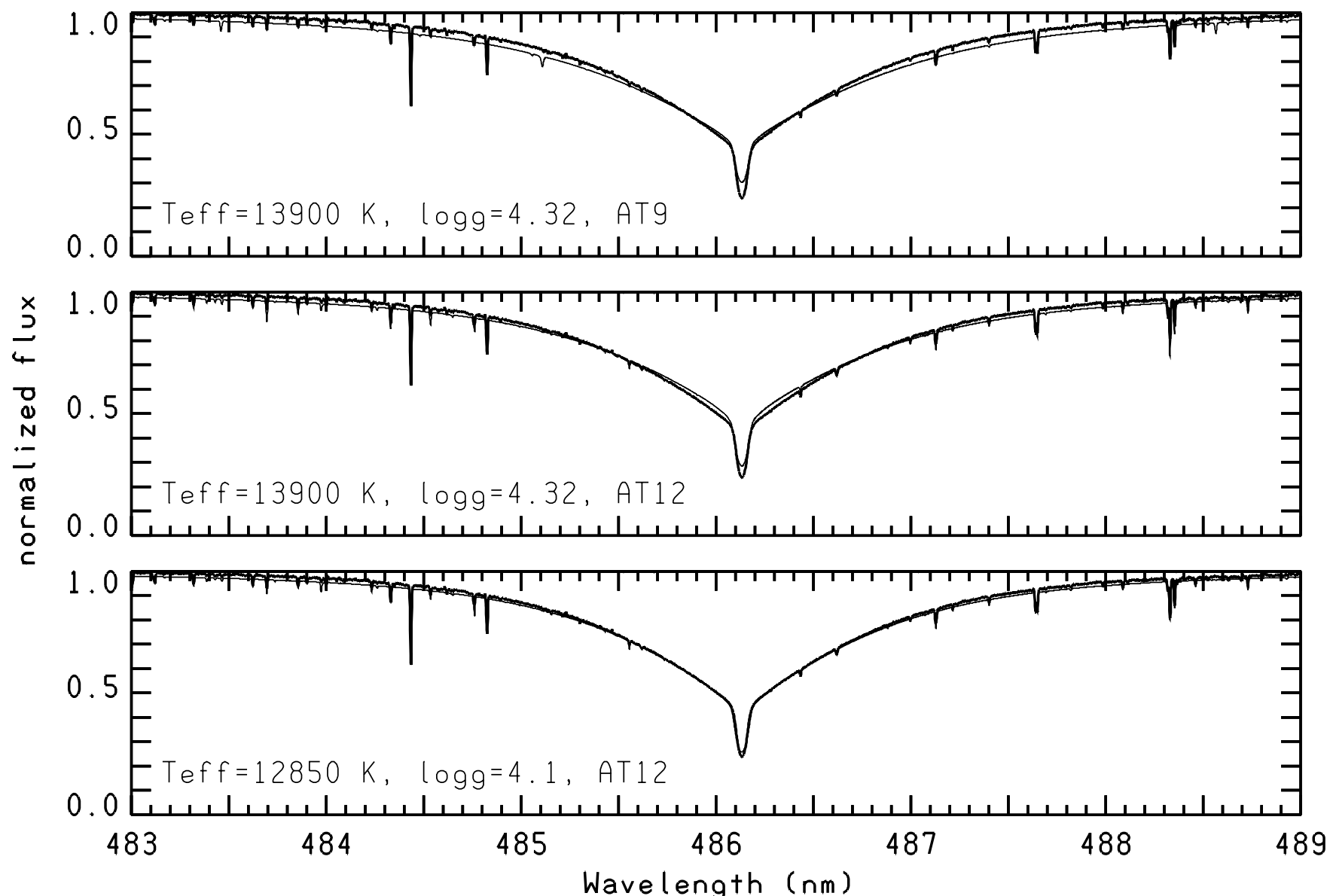

Fig. 2. The observed $\mathrm{H}_{\beta}$ (thick line) is compared to profiles computed with different models (thin lines). In the upper and middle panels the ATLAS9 model and the ATLAS12 model with parameters given by the photometry $\left(T_{\text {eff }}=13900 \mathrm{~K}, \log g=4.32, \xi=0.0 \mathrm{~km} \mathrm{~s}^{-1}\right)$ were used. The lower panel shows the profile computed with the final adopted ATLAS12 model with parameters $T_{\text {eff }}=12850 \mathrm{~K}, \log g=4.1$, and $\xi=0.0 \mathrm{~km} \mathrm{~s}{ }^{-1}$.

\section{The ATLAS12 model and abundance determination}

The opacity distribution function ATLAS9 model with parameters derived from the UBV and Strömgren photometry $\left(T_{\text {eff }}=13900 \mathrm{~K}, \log g=4.32,[\mathrm{M} / \mathrm{H}]=0.0, \xi=0.0 \mathrm{~km} \mathrm{~s}^{-1}\right)$ was used to derive preliminary abundances. Abundances for iron and phosphorus were obtained from equivalent widths, while those for all the other elements were obtained with the synthetic spectrum method. Lines listed in Castelli \& Hubrig (2004b) (electronic Appendix) were mainly examined. Synthetic spectra were computed with the SYNTHE code (Kurucz 1993). The adopted line lists are available on the web address given in the footnote ${ }^{4}$. The synthetic spectra were broadened for a rotational velocity $v \sin i=1.0 \mathrm{~km} \mathrm{~s}^{-1}$ and an instrumental resolving power of 80000 for $\lambda<4520 \AA$ and of 110000 for $\lambda>4765 \AA$ (Sect. 2). The adopted $v \sin i$ was that which reproduced best the observed profiles, in particular Mg II $4481 \AA$. It is in close agreement with the value of $0.0 \mathrm{~km} \mathrm{~s}^{-1}$ derived by Catanzaro et al. (2004).

With this model, the average abundance derived from the equivalent widths of $11 \mathrm{Fe}$ I selected lines was equal, within the errors limits, to that obtained from the equivalent widths of 34 selected Fe II lines, i.e. $\log \left(N(\mathrm{Fe} \mathrm{I}) / N_{\text {tot }}\right)=-3.65 \pm 0.06$ and $\log \left(N(\mathrm{Fe}\right.$ II $\left.) / N_{\text {tot }}\right)=-3.69 \pm 0.15$. At the same time, for

\footnotetext{
${ }^{4}$ http://wwwuser.oat.ts.astro.it/castelli/ linelists.html
}

$\xi=0.0 \mathrm{~km} \mathrm{~s}^{-1}$ there was no trend of the abundances versus the equivalent widths of the Fe II lines. The table with the measured equivalent widths and corresponding abundances is available at the website given in footnote 1 .

The abundances based on the ATLAS9 model were used as input data for an opacity sampling ATLAS12 model (Kurucz 1997; Castelli \& Kurucz 1994) computed for the same parameters of the ATLAS9 model. Due to the extremely peculiar abundances of HR 6000 , the T- $\tau_{\text {Ross }}$ structure of the ATLAS12 model is rather different from that of the ATLAS9 model, in particular in layers upper than $\log \left(\tau_{\text {Ross }}\right) \leq-0.5$. The comparison of the T- $\tau_{\text {Ross }}$ relations from ATLAS9 and ATLAS12 is similar to that shown in Castelli \& Hubrig (2004b). As a consequence, in spite of the better agreement achieved between observed and computed Balmer profiles, the Fe I-Fe II ionization equilibrium worsens. The average abundances for Fe I and Fe II became $-3.42 \pm 0.06$ dex and $-3.61 \pm 0.15$ dex, respectively.

A search for an appropriate ATLAS12 model suitable to reproduce both the Balmer profiles and the Fe I-Fe II ionization equilibrium has finally yielded, after several trials and errors, the parameters $T_{\text {eff }}=12850 \mathrm{~K}, \log g=4.1$, in good agreement with the values adopted by Catanzaro et al. (2004). The Fe I and Fe II abundances were $-3.89 \pm 0.06 \mathrm{dex}$ and $-3.88 \pm 0.16 \mathrm{dex}$, respectively. Figure 3 shows that this model also reproduces, for $E(B-V)=0.06$, the slope of the UV energy distribution given by the IUE images SWP14849 and LWR11431. However, a close inspection of Fig. 3 reveals the presence of some strong 


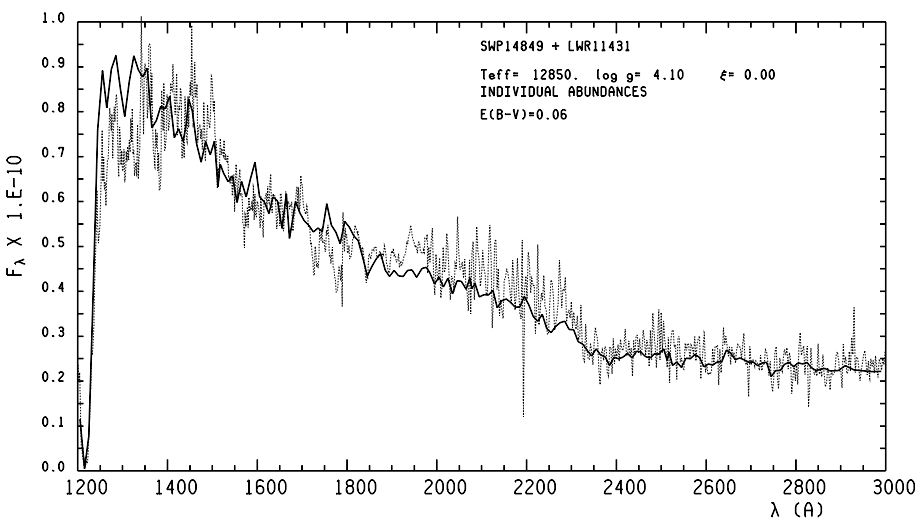

Fig. 3. Comparison of IUE data (dotted line) with the flux computed from the ATLAS12 model with individual stellar abundances (full line). Model parameters are $T_{\text {eff }}=12850 \mathrm{~K}, \log g=4.1$, and $\xi=0.0 \mathrm{~km} \mathrm{~s}^{-1}$. The IUE flux was dereddened for $E(B-V)=0.06$.

Table 2. Summary of the stellar parameters for HR 6000 from different determinations.

\begin{tabular}{llll}
\hline \hline Method & $T_{\text {eff }}$ & $\log g$ & Models \\
\hline$U B V \beta$ & $13973 \pm 300$ & $4.4 \pm 0.2$ & AT9 \\
$c_{0}, \beta$ & $13813 \pm 200$ & $4.3 \pm 0.05$ & AT9 \\
IUE flux & $13300 \pm 100$ & $4.1 \pm 0.1$ & AT9 \\
Balmer profiles & $12850 \pm 50$ & $4.1 \pm 0.1$ & AT12 \\
Fe I/Fe II & 12850 & 4.1 & AT12 \\
$V I_{\mathrm{c}} J$ & $11203 \pm 300$ & 4.0 (prefixed) & AT9 \\
\hline
\end{tabular}

absorptions in the short wavelength region which are neither predicted by the models nor detected in the examined IUE highresolution spectra (Sect.5.1). As already discussed in the previous section, the final ATLAS12 model fails to reproduce the observed Strömgren indices. The conclusion is that we were not able to fix a model well suited to reproduce the atmosphere of HR 6000 as deduced from the available observations. Table 2 summarizes the parameters from the different determinations based on the ATLAS9 (AT9) and ATLAS12 (AT12) models.

\section{The resulting abundances}

The abundances used for computing the whole synthetic spectrum of HR 6000 from $3050 \AA$ to $9460 \AA$ are listed in Col. 2 of Table 3 . The synthetic spectrum overimposed on the observed spectrum is available at the website given in footnote 1 . The abundances are based on the ATLAS12 model with parameters $T_{\text {eff }}=12850 \mathrm{~K}, \log g=4.1, \xi=0.0 \mathrm{~km} \mathrm{~s}^{-1}$, and $v \sin i=$ $1.0 \mathrm{~km} \mathrm{~s}^{-1}$. They are discussed briefly below.

The main results are the large underabundance of $\mathrm{Si}([-2.9])$ followed by that of $\mathrm{C}([-1.8])$. Other underabundant elements are $\mathrm{He}(\leq[-0.85]), N \leq([-1.7]), \mathrm{O}([-0.5]), \mathrm{Ne}([-0.9]$, $\mathrm{Mg}([-1.2]), \mathrm{Al}([-1.7]), \mathrm{S}([-1.5]), \mathrm{Cl}(<[-0.7]), \mathrm{Ca}([-0.2])$, $\mathrm{Sc}([-0.6]), \mathrm{V}([-1.1], \mathrm{Co} \leq([-0.8]), \mathrm{Ni}([-0.4])$, and $\mathrm{Sr}$ $([-1.0])$.

On the other hand, the most overabundant element is $\mathrm{Xe}$ $([+4.5])$, followed by $\mathrm{Hg}([+2.7])$, and $\mathrm{P}([+2.3]$ :). Other overabundant elements are Be $([+0.7]), \mathrm{Ti}([+0.25]), \mathrm{Mn}([+0.95])$, Fe $([+0.7]), \mathrm{Y}([+1.0])$, and possibly $\mathrm{Ba}([+1.0])$.

Below we discuss the individual abundances:

Helium: from the analysis of the He I lines $4713 \AA$ and $5875 \AA$, Catanzaro et al. (2004) derive an underabundance of
Table 3. Final abundances $\log \left(N_{\text {elem }} / N_{\text {tot }}\right)$ from the ATLAS12 model with parameters $T_{\text {eff }}=12850 \mathrm{~K}, \log g=4.1, \xi=0.0 \mathrm{~km} \mathrm{~s}^{-1}$. Solar abundances are from Grevesse \& Sauval (1998).

\begin{tabular}{lrrrr}
\hline \hline Elem & \multicolumn{1}{c}{ UVES } & IUE(old) & IUE(new) & \multicolumn{1}{l}{ Sun } \\
\hline $\mathrm{He}$ & -1.90 & & & -1.05 \\
$\mathrm{Be}$ & -9.90 & $\leq-9.5$ & -9.90 & -10.64 \\
$\mathrm{~B}$ & & -10.1 & -10.29 & -9.49 \\
$\mathrm{C}$ & $-5.32:$ & $\geq-5.4$ & -5.82 & -3.52 \\
$\mathrm{~N}$ & $\leq-5.82$ & $\geq-6.5$ & -6.50 & -4.12 \\
$\mathrm{O}$ & -3.71 & -3.3 & -3.70 & -3.21 \\
$\mathrm{Ne}$ & -4.86 & & & -3.96 \\
$\mathrm{Na}$ & -5.71 & & & -5.71 \\
$\mathrm{Mg}$ & -5.66 & -5.6 & -5.66 & -4.46 \\
$\mathrm{Al}$ & -7.30 & -7.7 & -7.30 & -5.57 \\
$\mathrm{Si}$ & -7.40 & $<-5.7$ & -7.40 & -4.49 \\
$\mathrm{P}$ & -4.30 & $-4.4--5.0$ & -5.00 & -6.59 \\
$\mathrm{~S}$ & -6.20 & $-6.0--5.5$ & -6.20 & -4.71 \\
$\mathrm{Cl}$ & $\leq-7.24$ & -4.3 & -7.24 & -6.54 \\
$\mathrm{Ca}$ & -5.88 & -5.2 & -5.88 & -5.68 \\
$\mathrm{Sc}$ & -9.50 & -11.0 & -9.50 & -8.87 \\
$\mathrm{Ti}$ & -6.77 & $>-6.5$ & -6.77 & -7.02 \\
$\mathrm{~V}$ & -9.14 & $-6.7--8.0$ & -9.14 & -8.04 \\
$\mathrm{Cr}$ & -6.37 & -6.2 & -6.37 & -6.37 \\
$\mathrm{Mn}$ & -5.60 & -5.3 & -5.60 & -6.65 \\
$\mathrm{Fe}$ & -3.85 & -3.8 & -3.85 & -4.54 \\
$\mathrm{Co}$ & $<-7.92$ & $\geq-9.3$ & -7.92 & -7.12 \\
$\mathrm{Ni}$ & -6.19 & -7.0 & -6.19 & -5.79 \\
$\mathrm{Cu}$ & $\leq-7.83$ & $\geq-9.3$ & -7.83 & -7.83 \\
$\mathrm{Zn}$ & & -8.3 & -7.44 & -7.44 \\
$\mathrm{Ga}$ & $\leq-9.16$ & -8.4 & -9.16 & -9.16 \\
$\mathrm{Sr}$ & -10.07 & & & -9.07 \\
$\mathrm{Y}$ & -8.80 & & & -9.80 \\
$\mathrm{Xe}$ & -5.40 & & & -9.87 \\
$\mathrm{Ba}$ & -8.919 & & -8.20 & -10.91 \\
$\mathrm{Hg}$ & -8.20 & - & -9.20 \\
\hline & & & &
\end{tabular}

[-1.45], i.e. $N(\mathrm{He}) / N_{\text {tot }}=0.0032$. We extended their analysis to all He I lines previously analyzed in the HgMn star HD 175640 by Castelli \& Hubrig (2004b), except for He I $4713 \AA$ which is located in a gap of the observed spectral regions. While all the He I lines were fairly well predicted in HD 175640 by the same abundance, this is not the case in HR 6000. In fact, the abundance $N(\mathrm{He}) / N_{\text {tot }}$ from the different lines ranges from 0.0125 to 0.002 , i.e from $[-0.80]$ to $[-1.60]$. The higher value reproduces the whole profile of He I $3867.4 \AA$ and only the wings of He I lines at $4026.1 \AA$ and $4387.9 \AA$, whose computed cores appear stronger than those observed (Fig. 4). The same discrepancy also occurs for the lines at $\lambda \lambda$ 4471.4, 4921.9, 5015.7 whose wings are fitted by abundances equal to 0.005 and 0.006 , while the cores would require a lower value. The two weak lines at $\lambda \lambda 5875.6$ and $6678 \AA$ are rather well predicted for $N(\mathrm{He}) / N_{\text {tot }}$ equal to 0.002 and 0.003 , respectively. Finally, the line at $7065.2 \AA$ is partially blended, both with telluric lines and with Fe II 7065.163 $\AA$. It cannot be reproduced whatever the abundance. The abundance from the remaining He I lines, which are very weak, depends on the assumed position for the continuum level. The comparison between observed and computed He I profiles is available at the website given in footnote 1.

Profiles of He I like those observed in HR 6000 were also observed in other HgMn stars, e.g. HR 7361, HR 7664 (Dworetsky 2004) or Feige 86 (Bonifacio et al. 1995). This kind of anomalous profiles was interpreted as evidence of helium stratification (Dworetsky 2004; Bohlender 2005). 


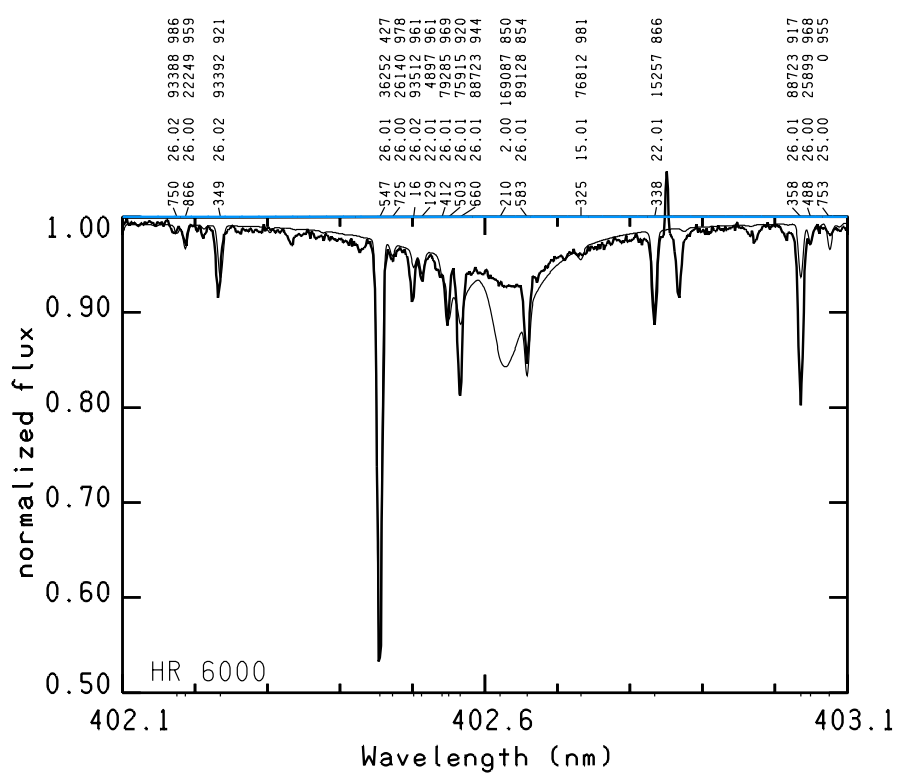

Fig. 4. Comparison of observed (thick line) and computed (thin line) He I $4026 \AA$ profiles. The abundance $\log \left(N(\mathrm{He}) / N_{\text {tot }}\right)=-1.90$ which fits the wings yields a computed core which is too strong.

No contribution of ${ }^{3} \mathrm{He}$ to $\lambda 6678 \AA$ was observed.

Beryllium: the abundance was derived from the lines at 3130.42 and $3131.06 \AA$, although the second one is heavy blended.

CNO: while Catanzaro et al. (2004) did not observe C II lines, we possibly identified very weak $\mathrm{C}$ II lines at $\lambda \lambda 3918.968$, 4267.01 , and $4267.26 \AA$. No nitrogen lines were observed. We deduced a carbon underabundance of $[-1.80]$ and an upper limit for nitrogen of $[-3.40]$. Oxygen is underabundant by $[-0.50]$. We examined all OI lines listed in Castelli \& Hubrig (2004b). The OI infrared lines at $\lambda \lambda 7774,8446$, and $9260 \AA$ are inadequately computed under the LTE assumption.

Neon: the abundance was fixed from Ne I 7032.413 $\AA$.

Sodium: there is a remarkable inconsistency between the abundance of -3.31 dex from the two lines Na I at $3302.368 \AA$ and $3302.978 \AA$ and the almost solar abundance of -5.57 dex of the NaI lines at $\lambda \lambda 5688.2,8183.2$ and $8194.8 \AA$. As already described by Catanzaro et al. (2004) the two stellar Na I D lines at $5889.951 \AA$ and $5895.924 \AA$ are completely hidden by the interstellar lines. The most likely explanation is that the lines at $3302 \AA$ are also of interstellar origin. They have been also observed in the spectrum of HD 175640 as weak red-shifted absorptions with the same wavelength displacement of $+0.08 \AA$ as the interstellar D-lines.

Magnesium: the estimated underabundance is $[-1.20]$. Magnesium abundance was mostly derived from Mg II $4481 \AA$. The Mg II doublet at 6545.942, 6545.994 $\AA$ is much broader than predicted and is also red-shifted. The most plausible explanation is the lack of Stark broadening data in the computations.

Aluminium: the estimated underabundance is $[-1.73]$ from the Al II lines at $\lambda \lambda 7042.06$ and $7056.60 \AA$. No other lines, as Al II at $\lambda \lambda 3944.009$ and $3961.523 \AA$, were observed.

Silicon: the estimated underabundance is $[-2.90]$. Only weak Si II lines at $\lambda \lambda 3853.665,3856.018$, and $3862.595 \AA$ were observed.
Phosphorus: the overabundance of phosphorus can be inferred from most of the P II and P III lines, but the real value is very difficult to deduce because the different lines give very different abundances. We measured the equivalent widths for a sample of $22 \mathrm{P} \mathrm{II}$ and $4 \mathrm{P}$ III lines. The average abundance from P II is $\log \left(N(\mathrm{P}) / N_{\text {tot }}\right)=-4.36 \pm 0.28$ for $\xi=0.0 \mathrm{~km} \mathrm{~s}^{-1}$, but the trend of the abundances with the equivalent widths is very large. It disappears for $\xi=6.0 \mathrm{~km} \mathrm{~s}^{-1}$. The corresponding abundance of $-4.70 \pm 0.12$ produces, for $\xi=6.0 \mathrm{~km} \mathrm{~s}^{-1}$, extremely broad and shallow profiles completely different from those observed.

The abundance for $\xi=0.0 \mathrm{~km} \mathrm{~s}^{-1}$ inferred from the P III lines is $\log \left(N(\mathrm{P}) / N_{\text {tot }}\right)=-4.57 \pm 0.27$. Therefore, the P II abundance for $\xi=0.0 \mathrm{~km} \mathrm{~s}^{-1}$ is 0.2 dex larger than that obtained from P III, but lies withing the large mean square error of the averages. The large uncertainty in the phosphorous abundance could be related to NLTE effects which have been neglected here. Another possibility could be the presence of phosphorus vertical abundance stratification.

The adopted P II and P III lines, together with the atomic data and the measured equivalent widths, are listed on the website of footnote 1 .

Sulfur: the sulfur underabundance of $[-1.5]$ was derived from the same lines used by Castelli \& Hubrig (2004b).

Chlorine: the absence of observed chlorine lines, such as Cl II 4794.54 A, implies an abundance less than -7.24 dex.

Potassium: potassium can be predicted by adopting solar abundances. However, the slight blue-shift of $-0.05 \AA$ of the lines K I $\lambda \lambda 7664.911,7698.974 \AA$, the only ones observed in the spectrum, may indicate an interstellar origin.

Calcium: all the Ca II lines listed in Castelli \& Hubrig (2004b) can be reproduced by an underabundance of [-0.2], except for the lines of mult. 13 (8201.720 $\AA$ and $8248.796 \AA)$ which are predicted to be too strong. The lines of the Ca II infrared triplet are red-shifted by $0.14 \AA$ and are discussed in more detail in Sect. 6.1.

The Ca I line at $4226.728 \AA$ is very weak. It is reproduced by solar abundance. A possible interstellar contribution can not be excluded.

Scandium: the underabundance of $[-0.6]$ was derived from the Sc II lines listed in Castelli \& Hubrig (2004b).

Titanium: the overabundance of $[+0.25]$ was derived from the Ti II lines listed in Castelli \& Hubrig (2004b).

Vanadium: the underabundance of $[-1.1]$ was deduced from the lines of V II mult. 1 at $3100 \AA$.

Chromium: most of the observed $\mathrm{Cr}$ II lines can be predicted by solar abundance.

Manganese: the abundance of $-5.0 \mathrm{dex}$ from the lines of Mn II mult. 3 at $3440-3498 \AA$ is larger by 0.6 dex than that obtained from most of the Mn II lines lying longward of the Balmer discontinuity. This result indicates the possible occurrence of vertical stratification for the abundance of this ion. The abundance of -5.6 dex listed in Table 3 is that obtained from the region longward of the Balmer discontinuity. Its value is the same as that presented by Catanzaro et al. (2004).

Iron: the abundance was derived from the equivalent widths of $11 \mathrm{Fe}$ I and $34 \mathrm{Fe}$ II lines. The lines together with the atomic data and the measured equivalent widths are available on the website of footnote 1 . For this paper, the oscillator strengths from Fuhr, Martin \& Wiese (1988) (FMW) were used. The overabundance of [+0.7] gives rise in the spectrum to a very large number of Fe I, Fe III, and especially Fe II lines (see Sect. 6.3). 
Cobalt: no Co II lines were observed in the spectrum, in particular the line at $3501.717 \AA$ is absent. Therefore only an upper limit of $[-0.8]$ was fixed.

Nickel: most Ni II lines indicate an underabundance of [-0.4].

Copper: no lines of $\mathrm{Cu}$ II were observed, in particular those at $\lambda \lambda 4909.734,4917.892$, and $4931.698 \AA$. Because for solar abundance there are no predicted lines we assumed solar abundances as upper limit for this element.

Gallium: no gallium lines were observed. If a feature at $5416.32 \AA$ is identified as Ga II it would be reproduced by $\log \left(N(\mathrm{Ga}) / N_{\text {tot }}\right)=-6.0$. This abundance gives rise to other strong computed Ga II lines with no observed counterparts.

Strontium: no Sr II lines were observed. Only a very weak absorption is detectable at the position of Sr II $4077.71 \AA$. It is reproduced by assuming an underabundance of [-1.0].

Yttrium: the overabundance of $[+1.0]$ was derived mostly from the Y II line at $4900.12 \AA$.

Zirconium: no $\mathrm{Zr}$ lines were observed in the spectrum. They are not predicted by assuming solar $\mathrm{Zr}$ abundance.

Xenon: Xenon is the most overabundant element ([+4.5]). The abundance was derived from the Xe II lines listed in Castelli \& Hubrig (2004b) having log $g f$ available from the NIST database ${ }^{5}$. They were taken from Wiese \& Martin (1980) (WM80). Furthermore, we added in the line list some lines with $\log g f$ 's from Ryabchikova \& Smirnov (1989). In addition, and only for identification purposes, all the lines with no $\log g f$ in the NIST database but with intensity $\geq 100$ were included in the line lists. A guessed $\log g f$ was assigned to them.

We noticed that the position of several Xe II lines is close to that of unidentified stellar lines with the wavelength blue-shifted by 0.05 to $0.1 \AA$ from the Xe II laboratory wavelength. Examples are the lines at $\lambda \lambda 4180.10,4208.48,4209.47,4238.25,4245.38$, $4330.52,4393.20,4448.13,4462.19,5667.56 \AA$. We have observed the same occurrence in the two Xe rich $\mathrm{HgMn}$ stars Feige 86 and 46 Aql (Castelli \& Hubrig, in preparation). If the Xe II identification is correct, the wavelength shift could be due to some isotopic anomaly. In fact, no isotopic composition was considered in our computations owing to the lack of isotopic wavelengths for Xe II. Data are only available for $\lambda 6051.150 \AA$ (Alvarez et al. 1979). For this line the isotopic wavelengths range from $6051.148 \AA$ to $6051.152 \AA$. Because the stellar line is blue-shifted by $0.03 \AA$ from the laboratory wavelength $6051.150 \AA$ (Hansen \& Persson 1987) an isotopic anomaly cannot be invoked to explain the observed shift.

Barium: we were not able to draw any conclusive results for barium. There is a weak absorption at $4933.93 \AA$, but if we identify it as Ba II $4934.076 \AA$, this barium line would be blue-shifted by $0.15 \AA$. The wavelength in the spectrum would correspond to the Ritz wavelength $4933.970 \AA$ rather than to the observed wavelength $4934.077 \AA$, as listed in the NIST database. Any isotopic anomaly has to be excluded because the shortest isotopic wavelength is that of the hyperfine component of ${ }^{137} \mathrm{Ba}$ at 4934.054 A (McWilliam 1998).

Mercury: only $\mathrm{Hg}$ II at $3984 \AA$ is observable. It can be predicted by assuming an overabundance of [+2.7] and a nonterrestrial isotopic composition. The $\mathrm{Hg}$ isotopic anomaly is discussed in Sect. 6.1.

5 http://physics.nist.gov/PhysRefData/ ASD/lines_form. html

\subsection{Comparison with the abundances from the IUE spectra}

Column 3 of Table 3 lists the abundances that were derived from IUE spectra by Castelli et al. (1985). The analyzed images were SWP04588 and LWR03979. We revised these abundances by using the same ATLAS12 model adopted for the analysis of the UVES spectra and the same images that we downloaded from the MAST archive ${ }^{6}$. Also updated line lists, as compared with those used by Castelli et al. (1985), were used. Column 4 of Table 3 lists the revised abundances from the ultraviolet.

The IUE spectra are very noisy and the resolving power is too low $(R=\sim 12000-13000)$ to allow an accurate analysis to be performed. Almost all very numerous lines are blended with either known or unknown components. In addition, the never adequately corrected distortions of the echelle orders make the drawing of the continuum a very problematic task. The large uncertainty in the continuum position affects both the shape and the strength of the spectral lines. For all these reasons the abundances that we derived from the IUE spectra have always been considered as estimates rather than definitive values.

Table 3 shows that the difference IUE(new)-IUE(old) between revised and Castelli et al. (1985) abundances is larger than 0.4 dex for $\mathrm{C}, \mathrm{O}, \mathrm{Cl}, \mathrm{Ca}, \mathrm{Sc} \mathrm{V}, \mathrm{Ni}, \mathrm{Zn}$, and $\mathrm{Ga}$. The abundances from UVES spectra reproduce the IUE spectrum (within all the above outlined limits) for all elements except carbon and phosphorous, for which the differences UVES-IUE(new) are +0.5 dex and +0.7 dex respectively. While the analysis of the carbon abundance from the visible spectral region is based only on very weak blended lines and therefore is rather uncertain, the phosphorous discrepancy confirms the difficulty in fixing the phosphorus abundance from the different lines.

The synthetic spectrum overimposed on the IUE spectra is available at the website of footnote 1 . It was computed with the abundances listed in Col. 4 of Table 3.

\section{Line spectrum peculiarities}

In the following sub-sections we discuss isotopic anomalies observed for $\mathrm{Hg}$ and $\mathrm{Ca}$ and the emissions observed for a few lines of Mn II, Cr II, and Fe II. We also discuss the huge quantity of lines which could not be identified.

\subsection{Isotopic anomalies}

Isotopic anomalies in HR 6000 were observed for the Ca II infrared triplet and for $\mathrm{Hg}$ II at $3984 \AA$. No ${ }^{3} \mathrm{He} /{ }^{4} \mathrm{He}$ isotopic anomaly was detected in the UVES spectra.

Ca II infrared triplet: the lines of the infrared triplet at $\lambda \lambda 8498.023,8542.091$, and $8662.141 \AA$ are red-shifted by $0.14 \AA$ and appear broader and stronger than those predicted. The wavelength red-shift of the infrared triplet observed in several CP stars (Cowley et al. 2007) was interpreted by Castelli $\&$ Hubrig (2004a) as due to a Ca isotopic composition different from the terrestrial one.

Hg II 3984: Hg II at $3984 \AA$, although weak, was detected in the UVES spectra. The isotopic composition that best reproduces the observed line for $\log \left(N(\mathrm{Hg}) / N_{\text {tot }}\right)=-8.20$ is that listed in Col. 3 of Table 4. For comparison, the terrestrial isotopic composition (Anders \& Grevesse 1989) is given in Col. 5 of Table 4. In HR 6000 the isotope 204 is the most abundant. The profiles computed with the stellar and terrestrial isotopic compositions

\footnotetext{
${ }^{6}$ http://archive.stsci.edu/iue/
} 
Table 4. Mercury isotopic (iso) and hyperfine (hfs) composition in \% for $\mathrm{Hg}$ II at $3984 \AA$.

\begin{tabular}{lc|rc|rcc}
\hline \hline Isotope & $\lambda$ & $\begin{array}{c}\text { stellar } \\
\%\end{array}$ & $\begin{array}{c}\log g f \\
\text { iso }\end{array}$ & \multicolumn{1}{|c|}{$\begin{array}{c}\text { terr. } \\
\text { \% }\end{array}$} & \multicolumn{1}{c}{ log $g f$} & $\begin{array}{c}\text { hfs. } \\
\%\end{array}$ \\
\hline 196 & 3983.769 & 0.15 & -2.81 & 0.15 & -2.81 & \\
198 & 3983.838 & 0.77 & -2.11 & 9.97 & -1.00 & \\
$199 \mathrm{a}$ & 3983.838 & 20.00 & -0.70 & 16.87 & -0.77 & 7.14 \\
$199 \mathrm{~b}$ & 3983.849 & 20.00 & -0.70 & 16.87 & -0.77 & 9.71 \\
200 & 3983.909 & 6.87 & -1.16 & 23.10 & -0.64 & \\
$201 \mathrm{a}$ & 3983.930 & 5.00 & -1.30 & 13.18 & -1.32 & 4.80 \\
$201 \mathrm{~b}$ & 3983.941 & 5.00 & -1.30 & 13.18 & -1.08 & 8.30 \\
202 & 3983.990 & 10.00 & -1.00 & 29.86 & -0.52 & \\
204 & 3984.071 & 57.20 & -0.24 & 6.87 & -1.16 & \\
\hline
\end{tabular}
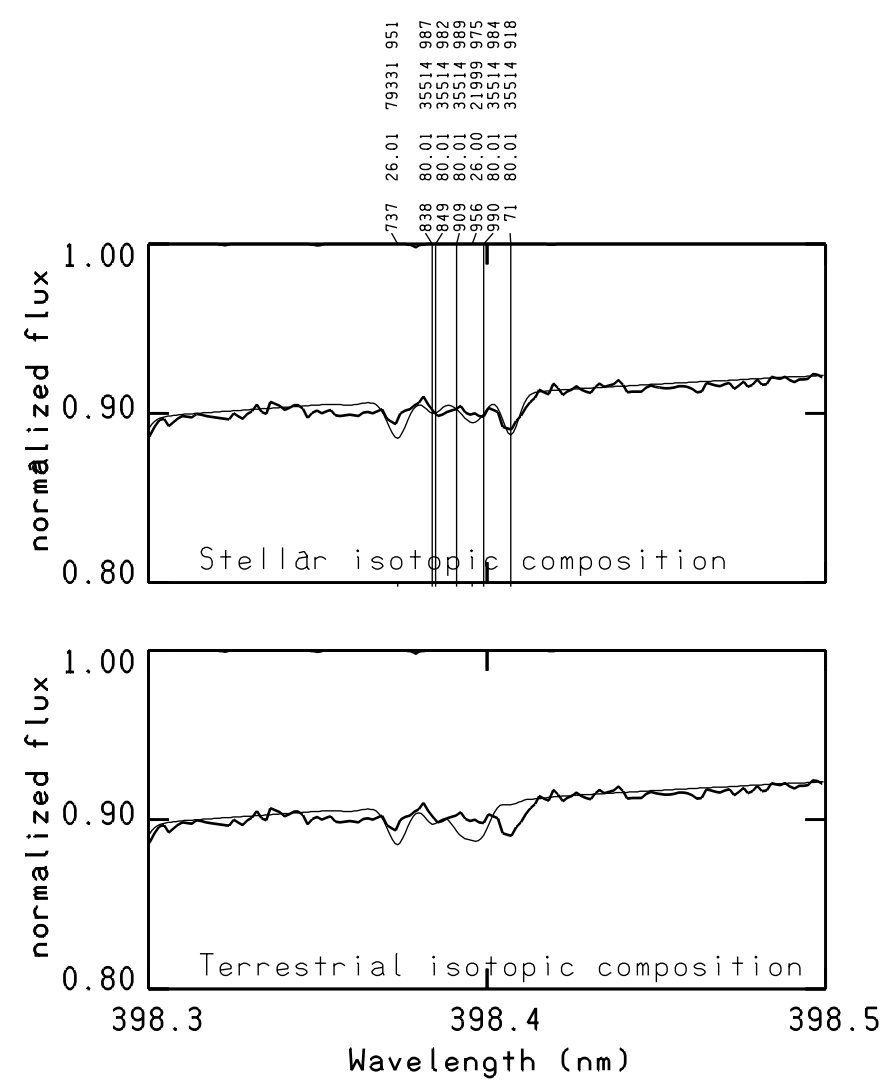

Fig. 5. The observed profile (thick line) of $\mathrm{Hg}$ II $3984 \AA$ is compared in the lower panel with the profile computed with a terrestrial isotopic composition (thin line) and in the upper panel with the profile computed with the isotopic mixture listed in Col. 3 of Table 4 (thin line).

are compared with the observed profile in Fig. 5. We note that the $\mathrm{Hg}$ II line is blended with Fe I $3983.956 \AA$.

Hg II at $1942.3 \AA$ can be observed in the IUE spectra, but the isotopic shifts are too small to enable us to confirm the isotopic composition deduced from the line at $3984 \AA$.

\subsection{Emission lines}

Emission lines were observed for $\mathrm{Mn}$ II, Cr II, and Fe II. We assumed that the studied lines are true emissions only if they were observed in both UVES spectra taken at two different epochs. Emission lines are listed in Table 5.

Emission lines of Mn II: some lines of Mn II multiplet 13 have been observed either in emission $(\lambda \lambda 6125.863$, $6126.218,6126.510,6131.923 \AA$ ) or much weaker than predicted $(\lambda \lambda 6122.434,6122.810,6128.734 \AA)$. Other lines $(\lambda \lambda 6129.033,6130.796,6131.016 \AA)$ are not observed in the spectrum taken on September 2005, while they appear as weak emissions in the spectrum of March 2006. This fact may suggest the presence of some variability in the emissions. Finally, the two very weak Mn II lines of mult. 13 at $6123.160 \AA$ and $6129.254 \AA$ are in absorption and agree well with the predicted lines.

Other weak Mn II emissions occur at 6446.337 (Mn II, mult. 19) which is blended with Fe II 6446.410 , and at $7219.968,8495.229,8565.819,8769.175 \AA$. An emission could also affect the line at $8695.208 \AA$, which is observed to be much weaker than predicted, and the line at $8819.584 \AA$ which, although predicted, is not observed. We note that there is quite good agreement for this last line between the observed and computed profiles in the HgMn star HD 175640 (Castelli \& Hubrig 2004b), even considering that no hyperfine structure is available for computations.

Emission lines of $\mathrm{Cr}$ II: a few $\mathrm{Cr}$ II lines arising from high excitation levels and having large log $g f$ 's appear clearly in emission. These lines were also found in emission in HD 175640 (Castelli \& Hubrig 2004b).

Emission lines of Fe II: there are no clear emissions for Fe II. The most probable ones are listed in Table 5. However, there is a set of observed lines much weaker than predicted. This disagreement could be explained either due to incorrect log $g f$ values or to emissions filling the absorptions. They occur at $\lambda \lambda 6145.611$, 6214.948, 6328.498, 6340.841, 6349.601, 6419.626, 6436.598, 6451.094, 6479.607, 6480.807, 6518.774, 6524.726, 6576.153, $6576.170 \AA$.

The phenomenon of weak emission lines at optical wavelengths for main-sequence B-type stars has been noted from observations of relatively few stars. Presently, explanations of this phenomenon have been put forward in the context of the non-LTE line formation (Sigut 2001) and possible fluorescence mechanisms (Wahlgren \& Hubrig 2000).

\subsection{Unidentified lines}

There is a huge number of unidentified lines in the spectrum of HR 6000. Their list is available at the webaddress given in footnote 1 . For instance, most impressive regions are $\lambda \lambda$ 4404-4411 $\AA$ and 5100-5300 $\AA$, which are overcrowded with unidentified absorptions. We estimate that most are due to Fe II. In fact, a comparison with the spectrum of HD 175640 (Castelli \& Hubrig 2004b) has shown unidentified lines at the same wavelengths also in this star, although less intense than in HR 6000, in accordance with the different iron abundances (Fig. 6). Therefore the unidentified lines are not a characteristic peculiar to HR 6000.

In the Kurucz line list for Fe II (Kurucz 2005) ${ }^{7}$ there are numerous transitions between observed levels with wavelengths close to those of the unidentified lines, but with such low $\log g f$ 's that none corresponding absorption is predicted. The comparison of $\log g f$ 's from Kurucz with the $\log g f$ 's from Raassen \& Uylings (1998) has shown agreement in the two determinations. Therefore, either both determinations are off by several orders of magnitudes or other transitions at the same wavelength occur. This possibility seems partly justified when the Kurucz line list including lines from both observed and

\footnotetext{
${ }^{7}$ http://kurucz.harvard.edu/atoms/2601/gf2601. pos
} 
Table 5. Emission lines observed in HR 6000.

\begin{tabular}{llrrrl}
\hline \hline$\lambda(\AA)$ & Element & Mult. & \multicolumn{1}{c}{$\log g f$} & \multicolumn{1}{c}{$\chi_{\text {low }}$} & Notes \\
\hline 5518.08 & $?$ & & & & \\
6112.906 & Fe II? & - & -3.753 & 90300.625 & not predicted \\
6122.434 & Mn II & 13 & 0.950 & 82136.400 & absorption filled by emission? \\
6122.810 & Mn II & 13 & 0.084 & 82136.400 & absorption filled by emission? \\
6125.863 & Mn II & 13 & 0.783 & 82144.480 & \\
6126.218 & Mn II & 13 & 0.230 & 82144.480 & \\
6126.510 & Mn II & 13 & -0.791 & 82144.480 & \\
6128.734 & Mn II & 13 & 0.588 & 82151.160 & absorption filled by emission? \\
6129.033 & Mn II & 13 & 0.208 & 82151.160 & variable? \\
6130.796 & Mn II & 13 & 0.354 & 82155.840 & variable? \\
6131.016 & Mn II & 13 & 0.053 & 82155.840 & blend with Fe II in absorption \\
6131.923 & Mn II & 13 & 0.053 & 82158.170 & \\
6158.621 & Cr II & - & 0.718 & 89174.080 & \\
6182.340 & Cr II & - & 0.452 & 89336.890 & \\
6446.337 & Mn II & 19 & -0.663 & 98423.300 & on the blue wing of Fe II 6446.410 A \\
6526.302 & Cr II & - & 0.173 & 89885.080 & \\
6585.241 & Cr II & - & 0.829 & 90850.960 & red-shifted \\
7174.368 & Mn II & - & 0.397 & 85960.460 & \\
7175.815 & Mn II & - & -0.023 & 85960.460 & \\
7219.968 & Mn II & - & 0.768 & 86057.440 & \\
7222.639 & Mn II & - & 0.252 & 86057.440 & \\
8495.229 & Mn II & - & 0.198 & 85960.460 & \\
8565.819 & Mn II & - & 0.278 & 86057.440 & \\
8695.208 & Mn II & - & 0.577 & 74560.010 & absorption filled by emission? \\
8740.349 & Fe II & - & 0.002 & 99068.450 & \\
8758.410 & $?$ & & & & \\
8769.175 & Mn II & - & 0.491 & 74560.010 & \\
8819.584 & Mn II & 13 & 0.347 & 74560.010 & absorption filled by emission? \\
8839.005 & Fe II & - & & & J78, unclassified \\
9020.127 & Fe II & - & & & J78, unclassified \\
9085.11 & $?$ & & & & \\
9208.4 & $?$ & & & & \\
\hline & & & & & \\
\hline
\end{tabular}

${ }^{a} \mathrm{~J} 78=$ Johansson (1978).

predicted levels is used ${ }^{8}$. In fact, numerous Fe II lines due to transitions between predicted levels have log $g f$ 's large enough to produce observable absorptions. Unfortunately, the uncertainty in their wavelength may be of the order of several Angstrom and also the corresponding $\log g f$ 's may be affected by rather large errors. Table 6 is an example of tentative line identification for the unidentified lines in the interval 5130-5136 $\AA$. As shown in Fig. 6 and in the lower panel of Fig. 7, out of 17 spectral lines displayed only three can be identified as arising from observed

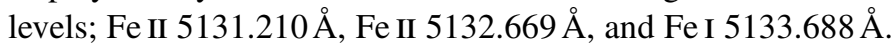
The middle panel of Fig. 7 shows that more lines would be identified if several $\log g f$ 's listed in Col. 5 of Table 6 are replaced by those given in Col. 7. They were derived by fitting the computed lines to the observed lines for the stellar iron abundance of -3.85 dex. Upper panel of Fig. 7 shows that the number of identified lines increases if the Fe II lines from predicted levels listed in Cols. 8 and 9 of Table 6 are added. The intensity of the lines from predicted levels is usually lower than that of the observed lines.

There is a remarkable coincidence between several Fe II lines measured by Johansson (1978) (Table 6, Col. 3) and the unidentified lines in our spectra. However, they are not predicted by the synthetic spectrum either because they are missing in the line lists or owing to their assigned log $g f$ 's which were too low.

\footnotetext{
${ }^{8}$ http://kurucz.harvard.edu/atoms/gf2601/ gf2601. lines 0600
}

\section{The T Tauri companion}

Van den Ancker et al. (1996) suggested the presence of a T Tauri companion for HR 6000 to explain the strong X-ray emission observed by Zinnecker \& Preibisch (1994) and the infrared excess that they inferred from the comparison of the observed energy distribution with that computed for the best fitting model having parameters $T_{\text {eff }}=14000 \mathrm{~K}, \log g=4.3$. They assumed a temperature of $3500 \mathrm{~K}$ for the T Tauri central star, an accretion rate of $2 \times 10^{-7} M_{\odot} \mathrm{yr}^{-1}$, and a $\mathrm{V}$ magnitude of about 13.5 , so that the companion would be about 6 mag fainter than HR 6000 and cannot contribute significantly to the observed visual spectrum. There are no clear spectroscopic traces of a second star in the spectrum of HR 6000, except for a broad weak absorption at $\lambda \lambda 6707-6708 \AA$ which, although of different intensity in the two spectra observed on September 2005 and March 2006, exhibits a very similar shape. The synthetic spectrum of HR 6000 considered as a single star is flat in this region except for two weak Fe II lines at 6707.232 $\AA$ and $6707.539 \AA$ which do not cover the whole observed absorption (Fig. 8, lower panel). We could argue that the feature is Li I 6707.7 which is a key element for the classification of T Tauri stars (Krautter et al. 1997). If such a star is present, it should be similar to a weak emission line T Tauri (WTT) star. WTT stars are defined as low-luminosity T Tauri stars with strong X-ray emissions and with an optical counterpart showing pre-main-sequence characteristics, in particular strong Li I absorption at $6707 \AA$ and very weak or also absent emissions in $\mathrm{H} \alpha$. Furthermore, the radial velocity of the WWT stars must 


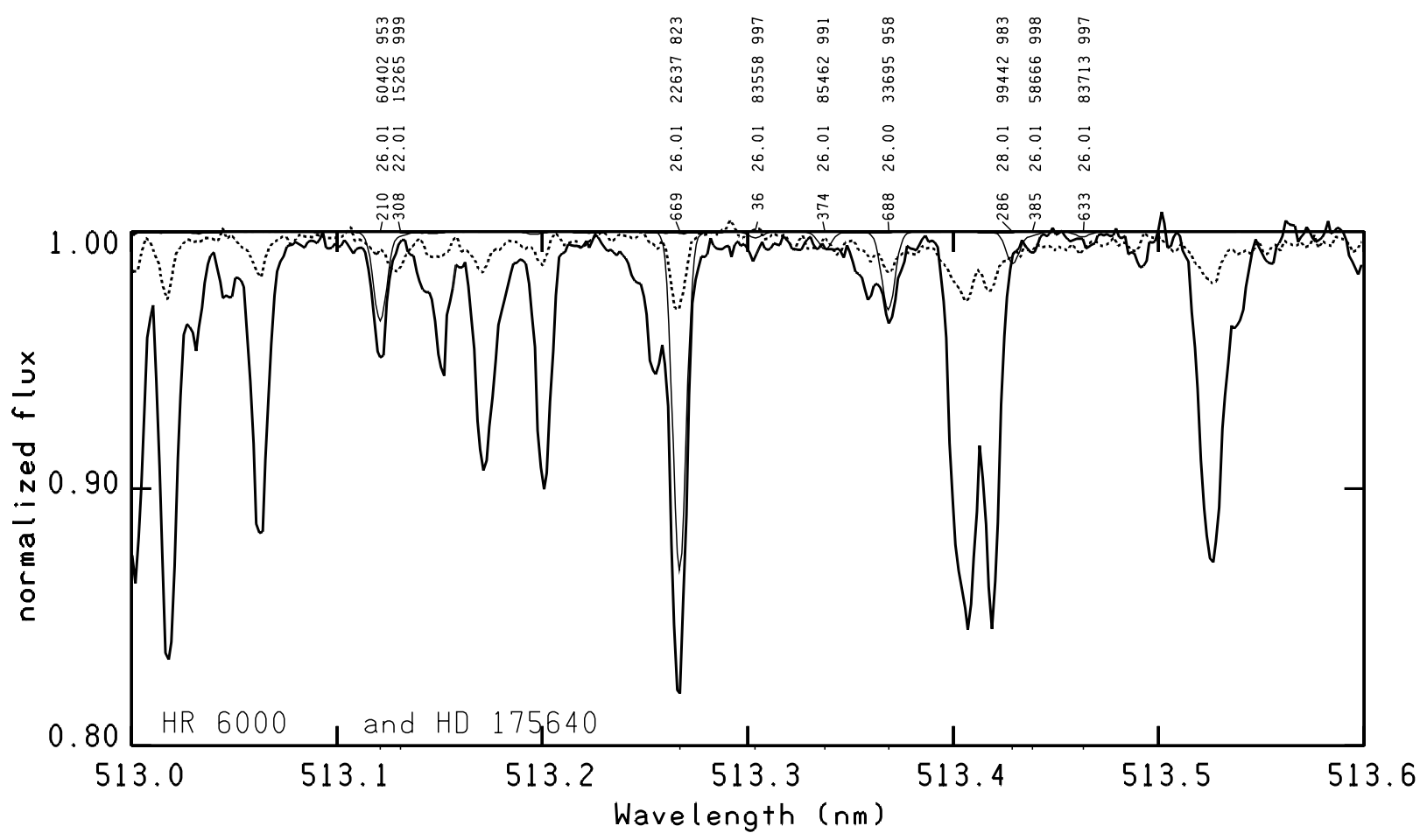

Fig. 6. The spectrum of HR 6000 (full thick line) is compared with that of HD 175640 (dashed line) in a region crowded with lines not predicted by the synthetic spectrum (thin line). The labels indicate the only identifications in this spectral range.

be consistent with that of the members of the molecular cloud to which they belong (Bertout 1989).

Upper panel of Fig. 8 shows that the observed spectrum is much better reproduced by a convolution of the synthetic spectrum of HR 6000 with a synthetic spectrum computed for $T_{\text {eff }}=3500 \mathrm{~K}, \log g=4.0$ and solar abundances. The computed spectrum of the cool star was not shifted in radial velocity relatively to that of $\mathrm{HR} 6000$. In this case the contribution of Li I 6707.7 improves the agreement between the computed spectrum and the observed spectrum whose continuum was lowered by $1.25 \%$. We note that the main contribution to the whole absorption comes from the $\mathrm{TiO}$ lines whose labels are not plotted in Fig. 8, due to their huge number. All the considerations relative to Fig. 8 can be extended to the whole spectrum, starting from about $5800 \AA$.

To model the combined spectrum shown in the upper panel of Fig. 8 we assumed a luminosity ratio $L_{\star} / L_{\text {TTauri }}=220$, which is among the values suggested by van den Ancker et al. (1996). When the distance $d=140 \pm 20$ pc adopted for HR 6000 by van den Ancker et al. (1996) is replaced by the Hipparcos distance $d=240 \pm 48 \mathrm{pc}$, the stellar luminosity computed with formula (1) from van den Ancker et al. (1996) is $L_{\star}=324 L_{\odot}$. In this case the luminosity of the T Tauri star becomes $L_{\text {TTauri }}=1.5 L_{\odot}$. For the computation of the binary spectrum the ratio of the stellar radii $\left(R_{\mathrm{TTau}} / R_{\mathrm{HR} 6000)}\right)^{2}$ was set to be equal to 0.8 in accordance with the Stefan-Boltzmann law. A change of the luminosity ratio affects only the level of the computed continuum. Several experiments made with other choices of the parameters with $T_{\text {eff }}$ ranging from $3500 \mathrm{~K}$ to $4500 \mathrm{~K}$ and $\log g$ ranging from 4.0 to 1.5 have shown that the above results are not significantly modified.

In any case, although we cannot exclude absolutely that the spectrum of HR 6000 is contaminated by that of a WTT star, neither can we exclude that we simply observe spectral noise rather than weak features of a secondary spectrum. In fact, the inspection of UVES spectra of other CP stars observed during the same runs has shown the presence of weak features very similar to those discussed here for HR 6000.

\section{Conclusions}

The present study of HR 6000 has led to the following results: the measured radial velocity indicates that HR 6000 most likely belongs to the Lupus cloud so that its age is of the order of $10^{7}$ years as was found for the Lupus 3 cloud (James et al. 2006). Interstellar lines from the cloud can be observed in the spectrum from the ultraviolet to the visible, with displacements of the order of $-0.05--0.15 \AA$ from the stellar lines.

UVES spectra observed with a 6 month time interval do not show spectrum variability for both stellar and interstellar lines, but a low variability of $1.2 \mathrm{~km} \mathrm{~s}^{-1}$ in the radial velocity and marginal variabilities of the weak spectral features which are however at the level of the noise.

Different methods for the parameter determination have led to large differences in $T_{\text {eff }}$. While the gravity values are found in the range between 4.1 and 4.4 dex with errors of the order of $0.1-0.2 \mathrm{dex}, T_{\mathrm{eff}}$ changes from $13900 \pm 300 \mathrm{~K}$, (if obtained from the $U B V \beta$ and uvby $\beta$ photometry), down to $12850 \pm 50 \mathrm{~K}$ when determined from the Balmer profiles, and can be as low as $11200 \pm 300 \mathrm{~K}$ when derived from the $V I_{\mathrm{c}} J$ photometry. Furthermore, as far as the Balmer profiles are concerned, the parameters which reproduce well $\mathrm{H}_{\beta}, \mathrm{H}_{\gamma}$, and $\mathrm{H}_{\delta}$ predict too narrow $\mathrm{H}_{\alpha}$ wings. All these discrepancies in the parameter determinations could be accounted for by the presence of the Lupus cloud with could affect the colors in such a way as to invalidate the standard reddening relations. Also a spectral contamination by a WTT companion, as suggested by van den Ancker et al. (1996), cannot be completely excluded, although the only spectroscopic sign of its possible presence is a weak variable broad 


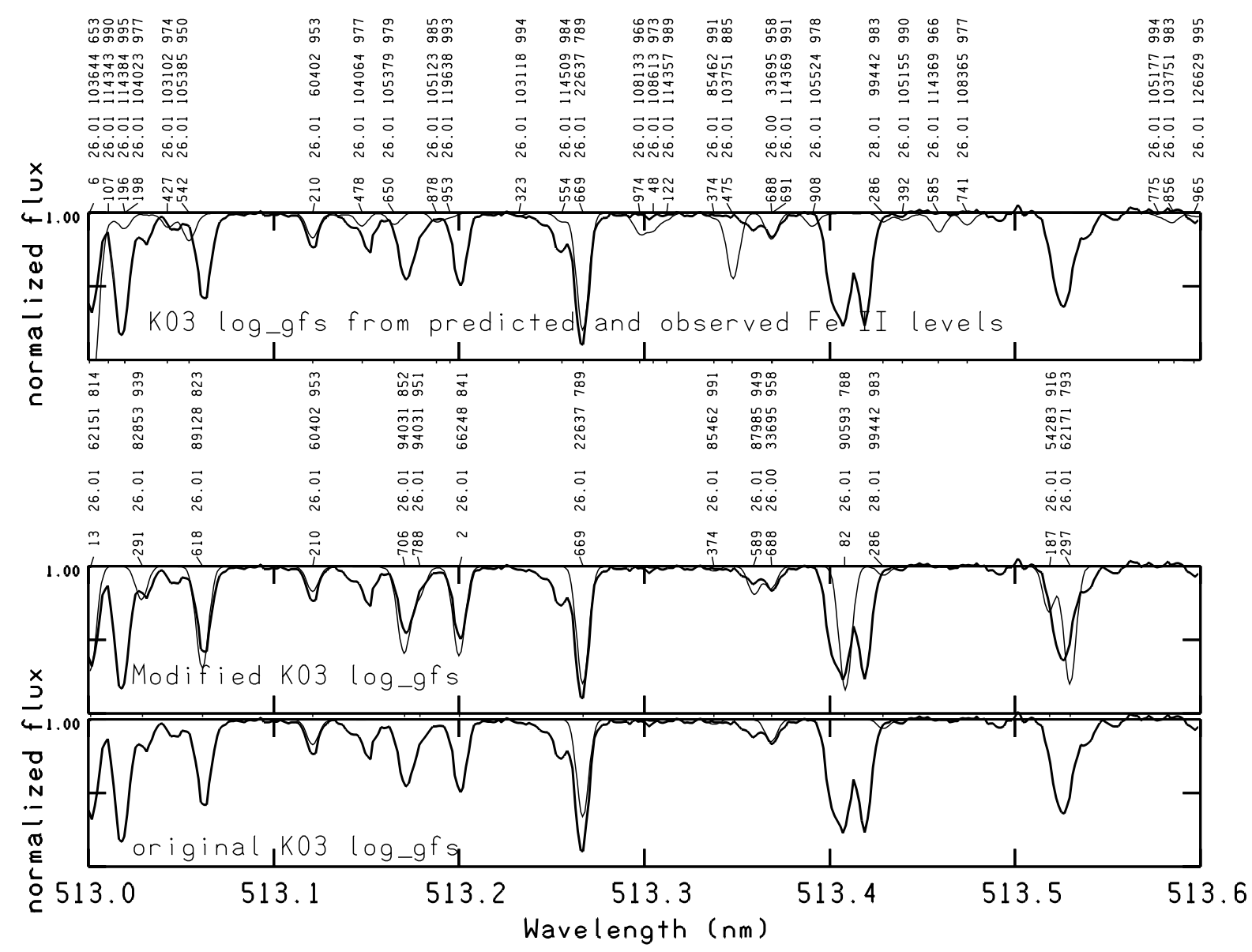

Fig. 7. The observed spectrum of HR 6000 (thick line) is compared with synthetic spectra (thin line) computed with the different choices of Fe II $\log g f$ 's. The lower panel shows the synthetic spectrum as predicted by the Kurucz (2003) line lists when only transitions from observed levels are considered. The middle panel shows the synthetic spectrum when modified log $g f$ 's for several Fe II lines are adopted (Table 6, Col. 7). The upper panel displays the synthetic spectrum computed by considering Fe II transitions from both observed and predicted levels.

absorption at the position of $\operatorname{Li}$ I $6707 \AA$ and a somewhat better agreement between observed and computed spectra longward of $6000 \AA$ for a combined synthetic spectrum obtained from that of HR 6000 and one computed for $T_{\text {eff }}=3500 \mathrm{~K}, \log g=4.0$, $[\mathrm{M} / \mathrm{H}]=0.0$. This companion could be either physically related to HR 6000 to form a close binary system or could be located in the foreground of HR 6000. Finally, the different parameters derived from the different determinations could be due to the extremely peculiar nature of HR 6000 which may make the classical model atmospheres inadequate to reproduce all the observed stellar quantities. In fact, the abundance stratification inferred for some elements, helium in particular, would require more refined models in which the hypothesis of constant abundances throughout the atmosphere is dropped.

Using an ATLAS12 model with parameters $T_{\text {eff }}=12850 \mathrm{~K}$, $\log g=4.1, \xi=0.0 \mathrm{~km} \mathrm{~s}^{-1}$ we have computed a synthetic spectrum for HR 6000 from $3050 \AA$ to $9460 \AA$ and have compared it with the observed spectrum. This comparison has shown that most of the elements lighter than $\mathrm{Ca}$ are significantly underabundant, except for $\mathrm{Be}, \mathrm{Na}$, and $\mathrm{P}$. The Si underabundance ([-2.9]) is remarkable because it is even lower than that of $46 \mathrm{Aql}([-1.0])$ which was claimed by Sadakane et al. (2001) to be the lowest found in $\mathrm{HgMn}$ stars.

A striking peculiarity of HR 6000 is the lack of any overabundance for heavy elements with $Z>40$, except for Xe and $\mathrm{Hg}$. The line spectrum of Xe II is similar to that we observed in a preliminary analysis of UVES spectra of $46 \mathrm{Aql}$, another xenon rich star: while most of the lines lie at the laboratory wavelength, some other Xe II lines seem to be shifted by about $-0.1 \AA$ from the predicted position. The same behaviour can also be observed in HD 175640, although to a lesser extent, owing to its lower Xe Overabundance. Unfortunately, the small number of known transition probabilities and the ignorance of the Xe II isotopic structure due to the lack of atomic data put strong limitations on the study of this element in the spectra of the CP stars.

The large overabundance of [+0.7] for iron generates a very rich line spectrum in which numerous, still unclassified Fe II lines are observed. A very large number of lines probably due to Fe II remain unidentified. The similar iron overabundance of $46 \mathrm{Aql}([+0.65])$ gives rise to an impressive close resemblance between the spectra of the two stars.

Abundances in the ultraviolet obtained from a re-analysis of the IUE spectra studied by Castelli et al. (1985) agree with abundances derived from the UVES spectra for all the elements, except for carbon and phosphorus. While the carbon identification in the optical spectrum is rather questionable owing to the weakness of the observed lines, the difference in the phosphorus abundance is similar to that yielded by the individual P II and P III lines.

There are several signs of vertical abundance stratification in HR 6000 for $\mathrm{He}, \mathrm{P}, \mathrm{Mn}$, and Fe. The peculiar shape of the He I profiles is discussed for the first time in this paper. 


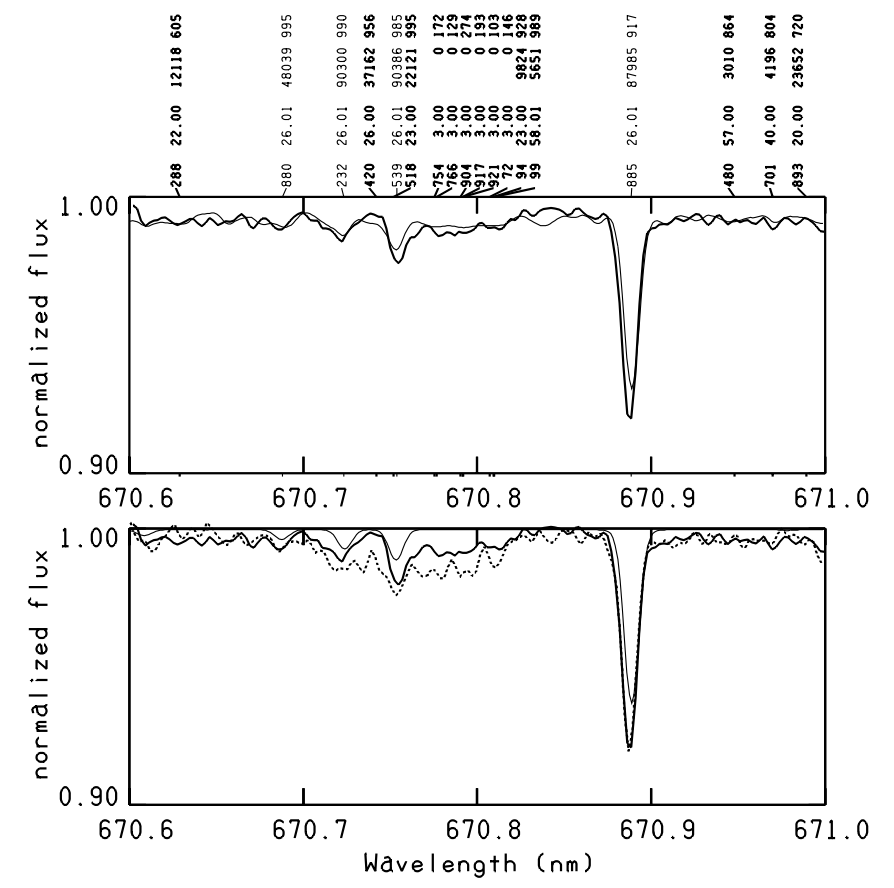

Fig. 8. Observed and computed profiles in the Li I region. The lower panel shows the spectra observed on September 2005 (thick line) and on March 2006 (dashed line) overplotted on the synthetic spectrum of HR 6000 considered as a single star (thin line). Upper panel compares the spectrum observed on September 2005 (thick line) with the spectrum computed by combining the synthetic spectrum of HR 6000 with a spectrum computed for $T_{\text {eff }}=3500 \mathrm{~K}, \log g=4.0,[\mathrm{M} / \mathrm{H}]=0.0$. The thin labels indicate atomic lines of HR 6000 and the thick labels those of the other star. No labels for molecular lines are printed, although molecular lines were considered for computing the synthetic spectrum of the cool star.

The profiles have cores too intense as compared to the wings, a fact indicating strong vertical $\mathrm{He}$ abundance stratification (Dworetsky 2004; Bohlender 2005). Furthermore, the inferred He abundance decreases with increasing wavelength ranging from an underabundance of [-0.8] at $4000 \AA$ to [-1.6] at $6000 \AA$.

For Mn II, the abundance shortward of the Balmer discontinuity is larger by about 0.6 dex than that longward of the Balmer discontinuity; for phosphorus, the P II abundance is larger by 0.2 dex than the P III abundance, but this discrepancy lies within the mean square error of the average abundances; for iron, the abundance from $\mathrm{Fe}$ II high excitation lines is generally higher by about 0.2 dex than that from Fe II low excitation lines.

Similar to other studied HgMn stars, also HR 6000 shows emission lines. The most numerous ones belong to Mn II, followed by those of $\mathrm{Cr}$ II and Fe II. While manganese and iron are overabundant in HR 6000, chromium has solar abundance. The star exhibits also isotopic anomalies for $\mathrm{Hg}$ and $\mathrm{Ca}$. In both cases the most heavy isotope is the predominant one.

Acknowledgements. We are very grateful to J. F. González for the re-reduction of UVES spectra using IRAF routines. and to Dr. J.V. Smoker for the useful discussion regarding interstellar lines.

\section{References}

Alvarez, E., Arnesen, A., Bengston, A., et al. 1979, Physica Scripta, 20, 141 Anders, E., \& Grevesse, N. 1989, Geochim. Cosmochim. Acta, 53, 197

Andersen, J., \& Jaschek, M. 1984, A\&AS, 55, 469

Andersen, J., Jaschek, M., \& Cowley, C. R. 1984, A\&A, 132, 354

Ballester, P., Grosbol, P., Banse, K., et al. 2000, SPIE, 4010, 246

Bertout, C. 1989, ARA\&A, 27, 351

Bessell, M. S., \& Eggen, O. J. 1972, ApJ, 177, 209

Bessell, M. S., Castelli, F., \& Plez, B. 1998, A\&A, 333, 231

Bohlender, D. 2005, EAS Publ. Ser., 17, 83

Bonifacio, P. 1989, NORMA: A Program for the Normalization of Spectra, University of Trieste, Astronomy Dept., Internal Rep. 20-Apr-1989

Bonifacio, P., Castelli, F., \& Hack, M. 1995, A\&AS, 110, 441

Castelli, F. 1999, A\&A, 346, 564

Castelli, F., \& Hubrig, S. 2004a, A\&A, 421, L1

Castelli, F., \& Hubrig, S. 2004b, A\&A, 425, 263

Castelli, F., \& Kurucz, R. L. 1994, A\&A, 281, 817

Castelli, F., \& Kurucz, R. L. 2004, [arXiv:astro-ph/0405087]

Castelli, F., \& Kurucz, R. L. 2006, A\&A, 454, 333

Castelli, F., Cornachin, M., Morossi, C., \& Hack, M. 1985, A\&AS, 59, 1

Catanzaro, G., Leone, F., \& Dall, T. H. 2004, A\&A, 425, 641

Comerón, F., Fernández, M., Baraffe, I., Neuhäuser, R., \& Kaas, A. A. 2003, A\&A, 406, 1001

Cowley, C. R., Hubrig, S., Castelli, F., González, J. F., \& Wolff, B. 2007, MNRAS, 377, 1579

Crawford, I. A. 2000, MNRAS, 317, 996

Crawford, D. L., \& Mandwewala, N. 1976, PASP, 88, 917

Dean, J. F., Warren, P. R., \& Cousins, A. W. J. 1978, MNRAS, 183, 569

Dworetsky, M. M. 2004, IAU Symp., 224, 727

Fuhr, J. R., Martin, G. A., \& Wiese, W. L. 1988, J. Phys. Chem. Ref. Data, 17, Suppl., 4

Grevesse, N., \& Sauval, A. J. 1998, Space Sci. Rev., 85, 161

Hansen, J. E., \& Persson, W. 1987, Phys. Scr., 36, 602

Hauck, B., \& Mermilliod, M. 1998, A\&AS, 129, 431

Hill, G. 1982, Publications of the Dominion Astrophysical Observatory Victoria, 16,67

James, D. J., Melo, C., Santos, N. C., \& Bouvier, J. 2006, A\&A, 446, 971

Johansson, S. 1978, Phys. Scr., 18, 217

Krautter, J., Wichmann, R., Schmitt, J. H. M. M., et al. 1997, A\&AS, 123, 329

Kurtz, D. W., \& Marang, F. 1995, Delta Scuti Star Newsletter, 8, 2

Kurucz, R. 1993, SYNTHE Spectrum Synthesis Programs and Line Data. Kurucz CD-ROM No. 18. Cambridge, Mass.: Smithsonian Astrophysical Observatory, 18,

Kurucz, R. L. 1997, The Third Conference on Faint Blue Stars, 33

Kurucz, R. L. 2003, http: //kurucz . harvard.edu/atoms/2601/ gf2601. lines 0600

Kurucz, R. L. 2005, Memorie della Societa Astronomica Italiana Supplement, 8, 86

Mathis, J. S. 1990, ARA\&A, 28, 37

McWilliam, A. 1998, AJ, 115, 1640

Moon, T. T. 1985, Commun. Univ. London Obs., 78

Raassen, A. J. J., \& Uylings, P. H. M. 1998, A\&A, 340, 300

Ryabchikova, T. A., \& Smirnov, Yu. M. 1989, Astron. Tsirk. N1534, 21

Sadakane, K., et al. 2001, PASJ, 53, 1223

Siebenmorgen, R., Prusti, T., Natta, A., \& Müller, T. G. 2000, A\&A, 361, 258

Sigut, T. A. A. 2001, ApJ, 546, L115

Smith, K. C. 1997, A\&A, 319, 928

Thé, P. S., \& Tjin A Djie, H. R. E. 1978, A\&A, 62, 439

Thé, P. S., Perez, M. R., Voshchinnikov, N. V., \& van den Ancker, M. E. 1996, A\&A, 314, 233

van den Ancker, M. E., de Winter, D., \& Thé, P. S. 1996, A\&A, 313, 517

Wahlgren, G. M., \& Hubrig, S. 2000, A\&A, 362, L13

Wiese, W. L., \& Martin, G. A. 1980, NSRDS-NBS, 68

Zinnecker, H., \& Preibisch, T. 1994, A\&A, 292, 152 
F. Castelli and S. Hubrig: A refined analysis of the remarkable Bp star HR 6000, Online Material p 1

\section{Online Material}


F. Castelli and S. Hubrig: A refined analysis of the remarkable Bp star HR 6000, Online Material p 2

Table 6. Unidentified lines in the region 5130-5136 ̊.

\begin{tabular}{|c|c|c|c|c|c|c|c|c|}
\hline $\begin{array}{c}\lambda(\mathrm{obs}) \\
(\AA)\end{array}$ & Identif. & $\begin{array}{c}\lambda \\
\mathrm{J} 78^{a} \\
\end{array}$ & $\begin{array}{c}\lambda \\
{\mathrm{K} 03^{b}} \\
\end{array}$ & $\begin{array}{l}\log g f \\
\mathrm{~K} 03^{b}\end{array}$ & $\begin{array}{c}\log g f \\
\mathrm{RU}^{c}\end{array}$ & $\begin{array}{c}\log g f \\
\text { Modified }\end{array}$ & \multicolumn{2}{|c|}{ predicted $(\mathrm{K} 03)^{b}$} \\
\hline 5130.0 & Fe II & 5130.011 & 5130.013 & -5.590 & -5.358 & -1.790 & 5130.006 & 1.172 \\
\hline 5130.18 & $\mathrm{Fe}$ II & 5130.177 & missing & & & & 5130.196 & -0.673 \\
\hline & Fe II & & & & & & 5130.198 & -0.573 \\
\hline 5130.3 & Fe II & & 5130.291 & -6.352 & & -1.252 & & \\
\hline 5130.45 & Fe II & & & & & & 5130.427 & -0.568 \\
\hline 5130.60 & Fe II & 5130.605 uncl. & 5130.618 & -6.012 & -5.562 & -0.312 & 5130.542 & -0.120 \\
\hline 5131.4 & Fe II & & & & & & 5131.478 & -0.570 \\
\hline 5131.5 & Fe II & & & & & & 5131.650 & -0.508 \\
\hline 5131.7 & Fe II & & 5131.706 & -5.127 & & -0.127 & 5131.878 & -0.727 \\
\hline & Fe II & & 5131.788 & -5.023 & & -0.723 & & \\
\hline 5132.0 & Fe II & & 5132.002 & -4.648 & -4.590 & -1.648 & 5131.953 & -0.222 \\
\hline 5132.55 & Fe II & & & & & & 5132.554 & -0.190 \\
\hline 5133.59 & Fe II & & 5133.589 & -3.961 & & -1.061 & 5133.475 & 0.220 \\
\hline 5134.07 & Fe II & & 5134.082 & -5.805 & & -0.105 & & \\
\hline 5134.2 & ? & & & & & & & \\
\hline 5134.9 & ? & & & & & & & \\
\hline 5135.25 & Fe II & 5135.268 & missing & & & & & \\
\hline & Fe II & & 5135.187 & -6.671 & & -2.671 & & \\
\hline & Fe II & & 5135.297 & -5.119 & -4.394 & -1.719 & & \\
\hline 5135.35 & ? & & & & & & & \\
\hline 5135.95 & ? & & & & & & & \\
\hline
\end{tabular}

${ }^{a}$ J78 = Johansson (1978).

${ }^{b}$ K03 = Kurucz (2003).

${ }^{c} \mathrm{RU}=$ Raassen \& Uylings (1998). 